\title{
Confocal microscopy: comparisons, applications, and problems
}

\author{
Claudette M. St. Croix ${ }^{1}$, Stuart H. Shand ${ }^{2}$, and Simon C. Watkins ${ }^{3}$ \\ ${ }^{1}$ University of Pittsburgh Graduate School of Public Health and ${ }^{2}$ University of Pittsburgh School of Medicine, Pittsburgh, PA, USA
}

BioTechniques 39:S2-S5 (December 2005)

doi 10.2144/000112089

\section{INTRODUCTION}

Over the past several years, fluorescence microscopy has become a primary quantitative tool in the armamentarium of the biomedical research scientist. In the past, the technology was limited to easily quenched fluorophores such as fluorescein and rhodamine, non-optimized objectives, filters consisting of little more than colored glass, and film as a recording media. At the present time, designer fluorophores span the entire visible spectrum to the near infrared, objectives are specifically designed to maximize throughput from fluorescent specimens, filters are made from specifically designed dielectric-coated glass, and modern cameras allow detection of vanishingly low numbers of photons such that a high-quality image may be recorded in a fraction of a second.

Improvements in confocal microscopy have paralleled the rapid advances in wide-field fluorescence microscopy. The goal of this review is a brief history and comparison of confocal technologies, as well as a few examples of the primary applications of confocal approaches.

Whereas confocal microscopes were initially only able to image one or two colors at relatively low speed, the modern confocal microscope is capable of collecting high-quality multispectral images across the visible range. The image shown in Figure 1 illustrates the high-power, high-resolution, and multicolor capabilities of the modern confocal microscope. Furthermore, specialized instruments work at very high speed and are thereby able to image living specimens with little photodamage. In this review we discuss the basic technology inherent to all confocal microscopes, the applications in which the confocal is an essential tool, with particular attention to the specialized use of confocal systems with in vivo

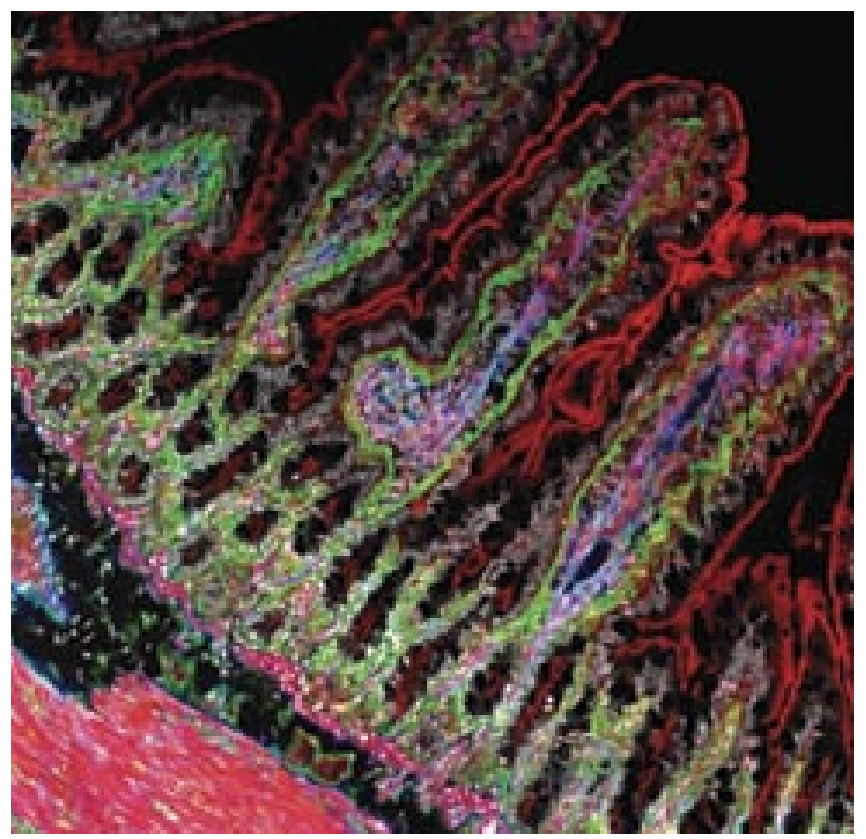

Figure 1. Confocal image of reconstructed rat ileum. Red, F-actin; white, nuclei; blue, smooth muscle actin; green, laminin. preparations, and provide a limited comparison of the applications of specific subtypes of confocal microscopes.

\section{THEORY AND INSTRUMENTATION}

When one looks at cells or tissues using wide-field fluorescence microscopy, the image consists of both in-focus light at the focal plane of the lens and out-of-focus light coming from other focal planes in the image. For example in Figure $2 \mathrm{~A}$, if one focused on the green plane, out-of-focus light from the red and blue planes would also be seen, thereby decreasing the signal-to-noise ratio in the final image. The confocal microscope essentially removes the out-of-focus light by inserting a pinhole at the image plane such that out-of-focus light does not reach the detector (Figure 2B). Only light focused at the pinhole passes through it, all other light is scattered.

The basic principle behind confocal microscopy is this use of spatial filtering to generate a focused spot of illumination. However, this solution only provides information about a single point at one time. In order to build an image, the focused spot of light must be scanned across the specimen. The first system, originally developed and patented by Marvin Minksy in the late 1950's (1), actually moved the stage under the illumination source. Subsequently, a number of confocal systems were described based on a similar design. These approaches, however, were extremely slow due to the mass of the stage. The first truly practical solution, described by White, Amos, and Fordham (2), was to scan the light beam over the specimen rather than moving the microscope stage. The core technology was to scan and collect light using two oscillating mirrors mounted on galvanometers. One mirror; the fast (line) scanning mirror, scanned in the $x$-axis, and the other (frame) mirror scanned more slowly in the $y$-axis. By coordinating the two scan axes, it was possible to build a complete image in a similar way to the way a television picture is generated. This confocal system was developed at the MRC Laboratory of Molecular Biology in Cambridge, UK and became the MRC 500 which in turn was marketed by Bio-Rad Laboratories (Hercules, CA, USA). This instrument and its several generations, was the market leading confocal microscope for a number of years. Numerous companies (i.e., Molecular Dynamics, Meridian, Noran, etc.), most of which have now gone out of business, also made variants on this design, generally called the point-scanning confocal microscope. Each was a modification on the theme, with the goal of making the device faster or having improved features over the current standard offerings. The primary microscope companies, Olympus (Melville, NY, USA), Nikon (Melville, NY, USA), Leica (Exton, PA, USA), and Carl Zeiss (Jena, Germany) also entered the fray, and all made fine instruments, some more successful than others. These early instruments, however, were limited by the number of colors that could be imaged and the computing and software that drove the instrument. In the current market, the pointscanning systems are now only made by the four primary microscope companies listed above. All manufacture very high-quality spectral, multichannel instruments that make confocal imaging simple, effective, and useful to the biomedical scientist with only a cursory knowledge of microscopic imaging. 


\section{Spectral Scanning}

The advent of fluorescent proteins as well as a continually expanding array of fluorophores with closely matched spectra has made the use of conventional barrier filters to separate emitted wavelengths a somewhat impractical solution. It is, for example, particularly challenging to separate emissions from the growing repertoire of genetically encoded fluorescent proteins that exhibit considerable spectral overlap. Fortunately, this problem has been solved within the past 4 years through the development and marketing of spectral detection systems. This instrumentation allows the precise separation of fluorescence signals with widely overlapping emission spectra by recording the spectral composition of fluorescence light in each of the scanned object spots. The four manufacturers of these devices have used differing approaches in their designs. Zeiss and Nikon use multi-element photomultiplier tubes (PMTs). The Zeiss detector currently collects eight spectral bins/scans (between 10 and $20 \mathrm{~nm} / \mathrm{bin}$ ), whereas the Nikon systems are able to collect a full spectral image (32 spectral bins simultaneously), In contrast, Leica, uses no barrier filters in the scan head at all, but separates light using monochromators. The spectral width of the monochromator can be changed, and sequential spectra collected. The Olympus systems use a combination of barrier filters and monochromators. The barrier filters select a range of light of each PMT, which may then be subselected using monochromators. In each case, the unmixing of spectral components dramatically improves signal separation. This technology has had a profound impact on the ability to separate images from closely matched fluorophores as well as allowing an easier approach to fluorescence resonance energy transfer (FRET) methodologies (see Applications section).

\section{Disk-Based Systems}

A key limitation in the use of point-scanning confocal microscopy for live cell imaging applications is the speed of image acquisition, which may be too slow to obtain information about rapid biological processes. Spinning disk systems provide an alternative means of obtaining a full-frame high-speed confocal image in real-time. The Nipgow disk, named after its inventor, Paul Nipgow (3), was developed as an attempt to encode pictures into radio signals using a pair of spinning metal disks impregnated with holes. Essentially, the arc-like array of pinholes scanned the image in a raster pattern, and the relative intensities of light were encoded as the disk passed over the image. This approach forms the core of the scan head devised by the Yokagawa Electric Corporation (Tokyo, Japan). The Yokagawa scan head improved on the relatively poor

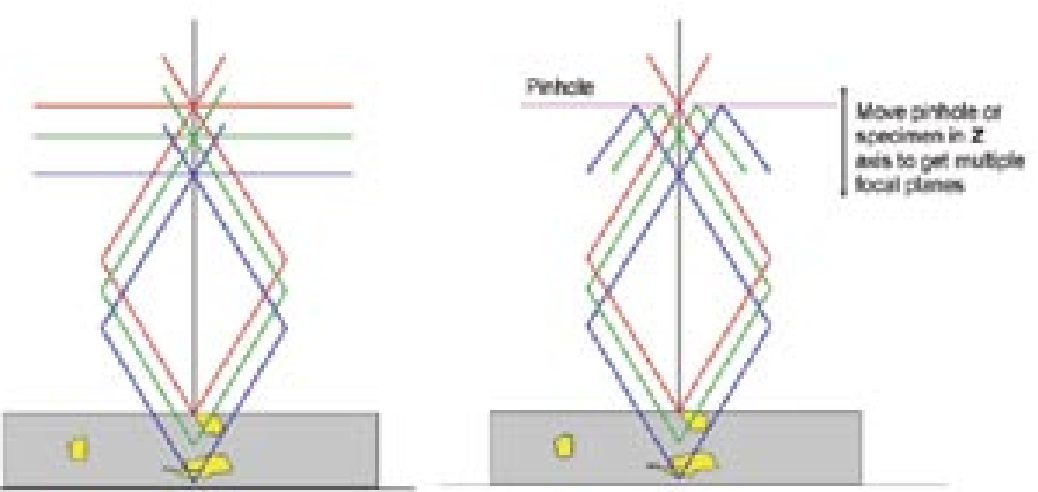

Figure 2. Principals of confocal microscopy. In wide-field fluorescence microscopy, the final image consists of light emanating from multiple focal planes (left panel) thereby decreasing the signal-to-noise ratio in the final image. The confocal microscope removes out-of-focus light by inserting a pinhole at the image plane (right panel). However, the pinhole will only provide information about a single point in space. It is therefore necessary to either move the specimen or move the exciting light to obtain the final image. light transmission of earlier designs by putting small lenses over each pinhole on the excitation side of the scan head to focus the illuminating light into the pinhole array. This very popular device is marketed by multiple companies, most notably by Perkin Elmer (Wellesley, MA, USA) as the UltraVIEW ${ }^{\circledR}$. Olympus recently introduced the DSU (Disk Scanning Unit) spinning disk confocal system that optimizes light transmission by incorporating a pattern of slits, rather than pinholes, on the disk. Both of these disk-based approaches use cameras for detectors rather than the PMTs used by the conventional point-scanning systems. While the disk-scanning systems do not produce the high-resolution images one can reliably get with point-scanning confocals, the speed of data acquisition is only limited by the read out rate of the camera being used.

Tremendous advances in camera technology in the last few years, most specifically the development of electron multiplying charge-coupled device (EMCCD) cameras, have had an enormous impact on the utility of the disk-scanning systems by improving signal-to-noise under very low light conditions and at high speeds of operation. As the EMCCD cameras may work at up to 30-40 $\mathrm{MHz}$, it is possible to collect more than $120512 \times 512$ images/s, which is extremely useful for fast live cell analyses. Furthermore, it is generally accepted that because of the minimal pixel dwell time of the illumination in these disk-based scan heads, photodestruction of the fluorophore and phototoxicity to the cell are greatly reduced. These are also major problems limiting the usefulness of traditional point-scanning systems for live cell imaging applications.

In the last year or so there have been two other other devices introduced that rely on point slit- or slit-scanning with lasers as well as linear CCD arrays or PMTs for detectors. Two very high-speed devices that are gaining significant interest come from Zeiss: (i) 5 Live, which uses a linear array CCD detector with a slit scan and (ii) Visitech, which uses a point slit approach with a PMT for detection.

\section{APPLICATIONS}

Fluorescence, or the generation of photons using controlled light sources and fluorophores, is inherently a quantum event. Lasers, particularly diode sources, are much more stable than standard mercury or xenon light sources, and as such, the confocal microscope is a highly quantitative tool. This is particularly so when one considers that the focal volume collected in each image is constrained by the pinhole size, the numerical aperture (NA) of the objective, and the wavelength of the emitted light. Apart from the pinhole size, which can be, and is, recorded by the software driving the microscope, these are constants. Therefore, as the imaging parameters can be maintained as a constant, the confocal microscope is capable of sampling and reporting intensity in single voxels. Importantly, this quantitation is highly reproducible. Variance in the imaged substrate is more likely to be due to variance in the sample than in the imaging system. The confocal microscope is therefore able to collect reproducible signals from specimens and reproducible z-axis slices with the only limitation being that, because of scattering and absorbance of light, the data should be sampled ideally at the same depth and in the same specimen. The confocal microscope can only penetrate and generate quantitative data for 20-50 $\mu \mathrm{m}$ in single photon mode, but when used appropriately, it is extremely quantitative. In this regard, the confocal microscope can be conceived as a flow cytometer, but with the added advantage of maintaining morphology so that cellular location can be correlated with signal intensity. This is an invaluable feature in examining the abundance of proteins or specific cell types within a specimen. 
One example would be in comparing cells within a tumor relative to those in the surrounding nonmalignant tissue.

\section{Reconstruction Technologies}

All modern confocal microscopes have motorized z-axis drives that permit precise optical sectioning of thick specimens. It is thereby possible to generate precise three-dimensional (3-D) data sets that can then be reconstructed into models of the sample in 3-D space. While this capability has been widely overused due to its visual appeal, it does provide structural information about the cell or tissue and its constituents and reveals detailed information regarding the localization of fluorescence within different compartments. For example, confocal microscopy has proven to be invaluable in understanding the complex structure and organization of neural tissue. High resolution images of primary neurons and dendrites labeled with fluorescent dyes can be used to reconstruct the intricate morphology of the neuron in 3-D space, such that quantitation of branching, arbors, etc., can be generated.

Multichannel confocal microscopy has also been used to describe the 3-D architecture of the mammalian brain, in particular the distribution and morphological relationship of the various types of neuronal and glial cells. Furthermore, time-lapse (4-D) confocal imaging of brain or spinal cord preparations can provide information about the dynamics of cell development and morphology in these tissues.

A specific example of the use of optional sectioning and 3-D reconstruction is in situ imaging of the blood vessels in the intact isolated perfused mouse lung (see Figure 3 and the Supplementary Movie available online at www.BioTechniques.com). Using confocal techniques, it is possible to visualize the vascular network, measure the number of vessels, the branch points, and the perfusion of individual vessels (when perfused with fluorescent beads). In addition, real-time measurements of cross-sectional area and maximal diameter can be generated using commercially available software, providing dynamic information about the physiological regulation of blood flow under a variety of experimental conditions.

\section{Co-Localization}

Co-localization analysis is used to determine whether or not two or more different molecules reside at the same physical location in a biological specimen. In the context of imaging, co-localization refers to the detection of emissions from two or more fluorescent molecules within the same pixel of the image. Using single optical sections with multicolor illumination and collection, it is possible to determine whether fluorescently labeled structures are located in the same lateral focal plane (z-slice). This co-localization is an association within the diffraction limited resolution of the confocal imaging system (approximately $200 \mathrm{~nm}$ in xy and $400 \mathrm{~nm}$ in z, depending on the NA of the objective and the wavelength of the emitted light). As this resolution is significantly less than the size of several important subcellular structures, such as coated vesicles, the confocal microscope can define whether proteins coexist within the same structure. It is not ideal, however, for larger structures such as endosomes and Golgi cisternae. In order to define precisely whether two fluorescent molecules are specifically associated, however, it is necessary to use FRET technologies in conjunction with confocal microscopy.
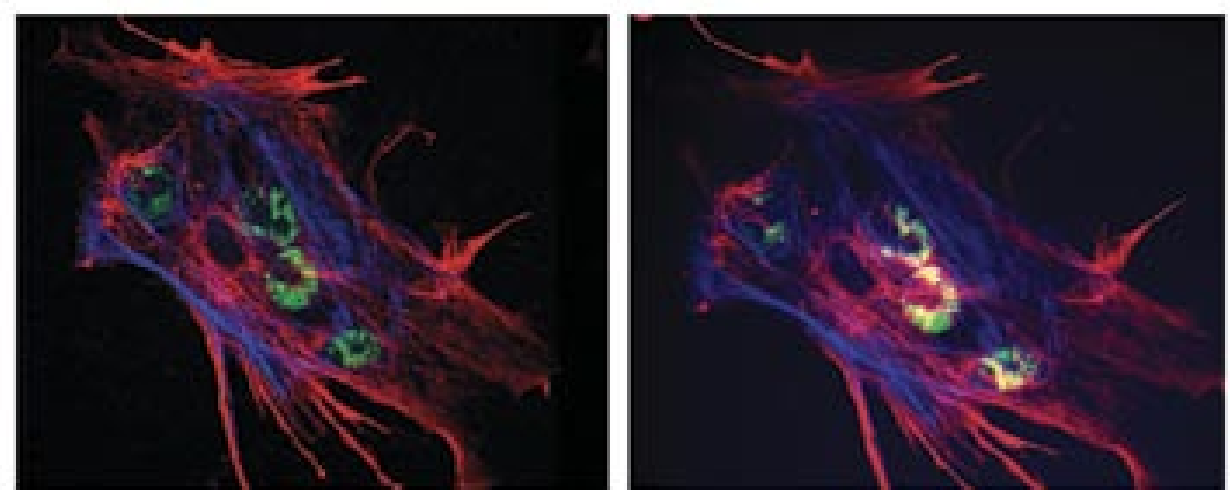

Figure 4. Confocal and wide-field imaging of hepatic stellate cells. Nuclei were labeled using cytox green (green). Actin filaments were visualized using $\mathrm{Cy}^{\mathrm{TM}} 3$-labeled phallodin (red), and tubulin staining is shown in blue. The left panel shows a data set taken with a confocal microscope (shown as a three-dimensional reconstruction). In the right panel, the wide-field image of the same cell is shown.

\section{Spectral Methods}

The presence of spectral capabilities in all the point-scanning confocals was described above. These massive data sets ( $x y z \lambda$ ) can be used to separate out very closely related spectra. This technology has significantly improved the accuracy of co-localization analysis by eliminating artifacts due to spectral bleed-through and autofluorescence. The use of spectral detectors has proven to be particularly invaluable in FRET-based applications, where the most commonly used donor and acceptor pairing 
(cyan and yellow fluorescent protein) exhibit significant spectral overlap, with $25 \%$ of the emission of the cyan bleeding into the yellow emission. Separation of cyan and yellow signals previously required the use of narrow detection bands and automatic switching of optical filters to differentiate between emissions, along with complex mathematical corrections to account for crosstalk between channels. Using spectral unmixing methods, the two components are separated with ease. Perhaps even more importantly, because these are point-scanning devices, it is extremely simple to place the laser beam over features within cells and selectively photobleach the acceptor in the FRET pair. This critical control verifies the occurrence of FRET, in both inter- and intramolecular applications, and is quite difficult to achieve with wide-field imaging systems.

\section{PROBLEMS WITH CONFOCAL}

Because the confocal microscope is now an extraordinarily easy tool to use, there is a tendency to misuse the instrument. For example, the axial thickness of optical sections depends on the inverse square of the NA. As such, when low NA, low magnification objectives are used, the optical section may be $7 \mu \mathrm{m}$ or more. As cryostat sections and many adherent cells are of this thickness or less, there is no rational reason for using the confocal microscope in these situations. Furthermore, confocal imaging requires a compromise between resolution, scan time, and photodestruction of the specimen. The higher the resolution, the more time required for the scan, and the longer the fluorophore is exposed to the laser. In many situations, enhancing resolution does not result in an increase in useful biological information about the specimen. This is illustrated in Figure 4. The left panel in Figure 4 shows a data set taken with a confocal microscope (shown as a 3-D reconstruction). In the right panel in Figure 4, the wide-field image of the same cell is shown. There is little difference in the two images, although the confocal image took $10 \mathrm{~s}$ to collect, whereas the wide-field image took $300 \mathrm{~ms}$ to collect.

\section{Phototoxicity and Photobleaching}

This latter point leads to perhaps the biggest problem with confocal microscope, the tendency to oversample both spatially and temporally in xy and $z$. It is commonly believed that increasing the laser intensity will increase the signal intensity. In fact, only a fraction of the available laser power is generally needed to maximally excite fluorophores in confocal microscopy. The remaining light will lead to quenching of the fluorophores, heating of the cell, and photooxidative damage to the cell. This is generally exacerbated by the tendency to oversample spatially. Because of this, point-scanning confocal microscopes should be used judiciously and carefully for live cell imaging applications. In fixed cell imaging, photobleaching will also occur when collecting serial z planes, or when multiple xy planes are collected and averaged. Thus it is extremely important when using a confocal system, to carefully consider the imaging parameters such that photodamage is minimized.

\section{SUMMARY}

The modern point-scanning confocal microscope is a highly quantitative tool, capable of collecting high-quality multispectral images across the visible range. Furthermore, the advent of spinning disk systems coupled with advances in camera technology has provided an alternative means of obtaining a full-frame confocal image at high speed. This approach is associated with marked reductions in the time the sample is exposed to the illumination source and is particularly useful for fast live cell analyses. While contemporary confocal systems are simple, effective, and very useful instruments, it is extremely important to (i) to judiciously manage the excitation light so that photodamage is minimized and (ii) to carefully consider whether confocal imaging is the appropriate approach for a particular biological application.

\section{ACKNOWLEDGMENTS}

The authors wish to acknowledge the technical expertise and contributions of Jason Devlin.

\section{COMPETING INTERESTS STATEMENT}

The authors declare no competing interests.

\section{REFERENCES}

1. Minsky, M. 1988. Memoir on inventing the confocal scanning microscope. Scanning 10:128-138.

2. White, J.G., W.B. Amos, and M. Fordham. 1987. An evaluation of confocal versus convential imaging of biological structures by fluorescence light microscopy. J. Cell Biol. 105:41-48.

3. Nipkow, P. 1884. Elektrisches teleskop [Electric telescope]. Berlin, Germany. Kaiserliches Patentamt, patent no. 30, 105.

Address correspondence to:

Claudette M. St. Croix

Department of Environmental and Occupational Health

100 Technology Drive

Cellomics Building, Rm 365

Pittsburgh, PA 15260, USA

e-mail: cls13@pitt.edu 